\title{
Objective analysis of intermediate-term outcome of the Ponseti technique: a review of the experience from Los Angeles
}

\author{
Lewis E. Zionts, Edward Ebramzadeh, Sophia N. Sangiorgio
}

The J. Vernon Luck, Sr., MD Orthopedic Research Center, Orthopaedic Institute for Children and the Department of Orthopedic Surgery, David Geffen School of Medicine at UCLA, Los Angeles, CA, USA

Contributions: (I) Conception and design: LE Zionts; (II) Administrative support: All authors; (III) Provision of study material or patients: All authors; (IV) Collection and assembly of data: All authors; (V) Data analysis and interpretation: All authors; (VI) Manuscript writing: All authors; (VII) Final approval of manuscript: All authors.

Correspondence to: Lewis E. Zionts, MD. The J. Vernon Luck, Sr., MD Orthopedic Research Center, Orthopaedic Institute for Children and the Department of Orthopedic Surgery, David Geffen School of Medicine at UCLA, 403 W. Adams Blvd, Los Angeles, CA 90007, USA. Email: LZionts@mednet.ucla.edu.

\begin{abstract}
The Ponseti method of manipulative treatment for clubfoot deformity became widely adopted by pediatric orthopaedic surgeons beginning in the mid-1990s. The technique allows correction of most idiopathic clubfeet using gentle manipulation and cast application. The treatment represents a marked advance over past efforts to gain correction of the foot through extensive release surgery. In 2006, we began a Clubfoot Clinic at the Orthopaedic Institute for Children in Los Angeles, California dedicated to managing clubfoot patients using Ponseti's method. An IRB-approved database of patient-related, treatment related, and demographic variables was assembled and used to ascertain the outcome of treatment as well as to address parental questions regarding certain aspects of treatment. Here, we present a review of our body of work, which has improved clinical decision making as well as our ability to better inform our patients' parents regarding the treatment and prognosis of the Ponseti method. Studies from our institution showed that while relapses and the need for extra-articular tibialis anterior tendon transfer (TATT) surgery remain common to the Ponseti method, these events do not adversely affect overall patient function or satisfaction. These findings were not unlike those of classic studies reported from Ponseti's institution. We conclude that the Ponseti method is not only a technique to achieve initial correction of an idiopathic clubfoot, but also how to manage relapses that will inevitably occur in many patients. While relapses and tendon transfer surgery are likely to remain common with this treatment method, these events do not adversely affect overall patient function or satisfaction. The parents of infants whose clubfeet are managed using the Ponseti method should be counselled accordingly.
\end{abstract}

Keywords: Idiopathic clubfoot; Ponseti method; treatment; deformity relapse; tendon transfer

Submitted Dec 02, 2020. Accepted for publication Mar 12, 2021.

doi: $10.21037 / \mathrm{atm}-20-7774$

View this article at: http://dx.doi.org/10.21037/atm-20-7774

\section{Introduction}

In 1948 Ignacio Ponseti developed a new form of manipulative treatment of idiopathic clubfoot deformity at the University of Iowa Hospitals and Clinics. His method was based on a firm understanding of the functional anatomy of the foot and ankle, and the response of the soft tissue and immature bone to redirection of mechanical stimuli (1). His technique consisted of a series of specific manipulations and cast applications to simultaneously correct the forefoot, midfoot, and subtalar components of the deformity; a percutaneous Achilles tenotomy was often necessary to correct the equinus component. Correction was maintained for the first few years using a foot abduction orthosis (FAO) at nighttime and during naps. 
Ponseti's recommendation for how long the FAO should be worn evolved over time. Specifically, in his earliest outcome study in 1963 (2), patients wore post-corrective braces for a mean total of 24.5 months: 3 months full-time, followed by an additional 21.5 months at nighttime. In the most recent study he co-authored in 2004, the FAO was used full-time for 2 to 3 months followed by nighttime use for 3 to 4 years (3). Considering these changes over time, it is not surprising that respondents to a 2010 survey of members of the Pediatric Orthopaedic Society of North American (POSNA) were equally divided as to their preferred duration of post-corrective bracing, with $23 \%$ recommending 2 years, $33 \%$ recommending 3 years, and $35 \%$ recommending 4 years (4).

The method described by Ponseti was not limited to achieving initial correction of the clubfoot, but also to the management of relapsed deformity. Ponseti observed that relapses, or recurrences, were common. He estimated that with very cooperative families who faithfully followed instructions, relapses could be prevented in about $50 \%$ of patients. In the other $50 \%$ a relapse will occur on average, around 2.5 years age $(2,5)$. He further noted that recurrences were rare in patients older than 5 years of age, and very rare after 7 years of age (1).

Ponseti believed that relapses resulted from the same pathology-retracting fibrosis of the muscle-tendon units and soft tissues in the affected limb-that caused the original deformity (6). Accordingly, he recommended the original correction be regained by further manipulations and cast applications using the same technique used to correct the original deformity. A repeat tenotomy was recommended if $15^{\circ}$ of ankle dorsiflexion could not be regained by cast treatment alone. Once the foot was wellcorrected in younger infants, bracing was resumed. In patients older than 2.5 years of age, he recommended transfer of the tibialis anterior tendon transfer (TATT) to the third cuneiform, if this muscle strongly supinated the foot, to prevent further relapse. He asserted a tendon transfer should only be performed after the foot was wellcorrected by cast treatment (5). Open release of the joints was discouraged due the propensity of such procedures to lead to stiffness, limited function, and pain in adult life $(1,7)$.

Ponseti cautioned that achieving complete anatomic correction was not to be expected, particularly in very severe, stiff clubfeet in which full reduction of the medially rotated navicular and full abduction of the calcaneus to its normal position were not possible. However, he noted the resulting range of motion of the foot and ankle was "sufficient for the normal activities into the fourth decade" and possibly beyond (1). Accordingly, his stated goal of treatment was to achieve a functional pain-free, normal-appearing, plantigrade foot, without calluses, and the ability to wear normal shoes (1).

Presently, the Ponseti method has gained wide acceptance among pediatric orthopaedic surgeons. In an article we coauthored in collaboration with the American Academy of Orthopaedic Surgeons using data from the Centers for Disease Control and Prevention and the Nationwide Inpatient Sample, we reported a decline in the rate of extensive surgery for the treatment of idiopathic clubfoot in patients less than 12 months of age in the United States between 1996 and 2006 (8). In a later 2010 survey of POSNA members, we reported $96.7 \%$ of respondents used the Ponseti method to manage idiopathic clubfoot deformity, with the majority adopting the method around the year 2000 (9).

\section{Background}

In July 2006, we established a Clubfoot Clinic at the Orthopaedic Institute for Children in Los Angeles dedicated to the treatment of clubfoot deformity closely adhering to the principles outlined by Ponseti. Our clinic is in an urban setting, with an affiliation to a nearby university hospital.

The families of all patients with an idiopathic clubfoot deformity receiving treatment at our clubfoot clinic were invited to participate in an IRB-approved database study. Patient-related variables including, sex, initial severity (graded with the Diméglio criteria) (10), and laterality were included. Demographic variables, including parental marital status, education level, insurance type, and family income were collected. Treatment-related variables, such as the number of casts applied prior to an Achilles tenotomy (if needed) to obtain initial correction, family-reported adherence with bracing, relapse(s) of deformity, and any subsequent treatment of relapse(s) were recorded. Dates of onset of treatment, latest follow-up, and onset of walking were also recorded. Date of brace discontinuation, reason for discontinuation, date of Achilles tenotomy (and repeat tenotomies), if performed, and date of TATT, if performed, were recorded.

Outcome was assessed at latest follow-up using the Dallas criteria described by Richards et al. (11) as good (a plantigrade foot with, or without, a tenotomy), fair (a plantigrade foot that had a limited posterior release or tibialis anterior transfer), or poor (a foot that had a 
posteromedial surgical release). Additionally, beginning at 3 years of age, the Roye Disease-Specific Instrument (DSI) (12), which consists of 10 items designed to assess overall satisfaction, appearance, pain, and physical limitations, was collected annually. In total, our database included more than 200 variables for each patient, excluding individual radiographic measurements and other parameters acquired for specific studies.

\section{The Ponseti method as practiced at our facility}

\section{Initial correction}

As recommended by Ponseti (1), the task of manipulating clubfeet and applying casts was not delegated to unsupervised assistants, rather they were performed or carefully supervised by the senior author (LEZ). We observed that initial correction of most idiopathic clubfeet could be obtained by 4 to 6 manipulations to correct the tarsal displacements, each followed by a cast application; an Achilles tenotomy was done in approximately $90 \%$ to $95 \%$ of patients to correct the equinus component of the deformity $(13,14)$.

We made some minor modifications to Ponseti's recommendations to best accommodate our patients' families, our hectic clinic, and limited resources at our facility. For example, while Ponseti urged the use of plaster of Paris casting material due to mouldability, we chose to use semirigid fiberglass cast material. This decision allowed parents who did not have ready access to an emergency room or urgent care facility to easily remove the casts if they became concerned about their infant's discomfort or circulation to the toes. The effectiveness of using semirigid fiberglass cast material has been documented by other authors (15-19). Another example pertains to percutaneous tenotomies. Specifically, in his original outcome study, Ponseti performed his percutaneous Achilles tenotomy procedures under general anesthesia (2); however he later urged the procedure be done under local anesthesia (20) in a clinic setting. Due to our sizeable clinic volume, we elected to do our Achilles tenotomies under conscious sedation as an outpatient procedure in an operating room. We found this procedure could be safely performed using propofol sedation without the need for airway instrumentation (21). The safety and efficacy of performing a tenotomy under anesthesia has been reported by other centers $(22,23)$. The anesthesia protocol used at any institution should always depend on the experience of the anesthesiologist and surgeon.

Following removal of the final cast, we used the FAO designed by Mitchell and Ponseti to maintain correction of the deformity (14). We instructed parents to place the infant in the brace for 23 hours per day for 3 months and, thereafter during naps and at nighttime. Families were urged to continue bracing for a minimum of 4 years. If the patient experienced a relapsed deformity during the first 4 years or was initially assessed to have a very severe deformity, bracing was recommended until the child reached 5 years of age.

The patients were carefully followed to assess brace fit and examined for early signs of relapsed deformity. Once the brace was applied, the patients were generally followed every 3 months until age 2 years, then every 4 months until age 4 years, every 6 months until age 5 years, and annual visits thereafter. At each visit we evaluated parental adherence to brace recommendations, proper fit of the FAO, condition of the skin over both feet, and general alignment of the forefoot and heel. Additionally, we measured passive ankle dorsiflexion and the thigh-foot angle to detect any changes from the prior visit that would suggest early signs of relapse. We also observed the patient while walking to determine the presence of inward deviation of the foot to the forward line of progression, dynamic forefoot supination during swing phase, or increased contact of the lateral boarder of the foot during stance phase; findings indicative of a supinating influence on the foot by the tibialis anterior muscle.

\section{Management of relapsed deformity}

A relapse was considered present if there was any recurrent deformity-usually consisting of slight equinus and varus deformity of the heel-which was deemed to require further cast treatment or surgery. In general, a relapse seen in a young infant was managed by a series of one to three manipulations and long leg cast applications using the same technique as that used originally to treat the foot. Once correction was regained, use of the brace was resumed. If a child older than 2.5 years of age had a relapsed deformity, the family participated in shared decision making with the option to resume use of the FAO or undergo TATT, especially if the child had experienced multiple prior relapses, or if dynamic forefoot supination was present $(1,24)$.

Prior to TATT, correction of the foot was regained by cast treatment. If $10^{\circ}$ to $15^{\circ}$ of dorsiflexion could not be achieved by cast treatment (25), the Achilles tendon 
Table 1 Percentage of clubfeet that relapsed, had an ATTT preformed, in mid to long-term outcome studies

\begin{tabular}{|c|c|c|c|c|}
\hline Study [year] & Relapses (\%) & ATTT (\%) & Outcome: feet (\%) & $\begin{array}{l}\text { Age at follow- } \\
\text { up (years) }\end{array}$ \\
\hline Ponseti and Smoley [1963] (2); n=94 feet & $53 / 94(56 \%)$ & $39 / 94(41 \%)$ & $\begin{array}{l}\text { Good: } 67(71 \%) \text {; acceptable: } 26(28 \%) \text {; } \\
\text { poor: } 1(1 \%)\end{array}$ & 5 to 13 \\
\hline $\begin{array}{l}\text { Zionts et al. [2018] (13); n=147 feet; } 101 \\
\text { patients }\end{array}$ & $101 / 147$ (69\%) & $56 / 147$ (38\%) & $\begin{array}{l}\text { Good: } 63(62 \%)^{\star} \text {; acceptable: } 38(38 \%)^{*} \text {; } \\
\text { poor: } 0^{\star}\end{array}$ & 5 to 10 \\
\hline $\begin{array}{l}\text { Cooper and Dietz [1995] (32); n=71 feet; } \\
n=45 \text { patients }\end{array}$ & $\mathrm{N} / \mathrm{S}$ & $38 / 71(54 \%)$ & $\begin{array}{l}\text { Excellent } 28(62 \%)^{*} \text {; good } 7(16 \%)^{*} \text {; poor } \\
10(22 \%)^{*}\end{array}$ & 25 to 42 \\
\hline
\end{tabular}

*, patients. ATTT, anterior tibial tendon transfer; N/S, not stated.

was lengthened. The tendon transfer was done using two incisions. We transferred the whole tendon from its insertion at the base of the first metatarsal into the third cuneiform without changing its position beneath the extensor retinaculum. The procedure has been described previously $(1,25,26)$.

Studies of the mid-term outcome of TATT have demonstrated the procedure to be effective for correcting dynamic forefoot supination and maintaining clinical improvement of ankle dorsiflexion and subtalar eversion (27-29). Similarly, plantar pressure testing of patients who had undergone this procedure showed both better foot alignment and improved pressure distribution throughout the foot (30,31). More importantly, satisfactory longterm clinical outcomes of this procedure have been documented $(32,33)$.

\section{Summary of our clinical research}

\section{Outcome of treatment}

The early outcome of the Ponseti method at our clubfoot clinic was first evaluated in 57 patients (84 clubfeet) treated between September 2006 and June 2008, with a minimum of 2 years of follow-up (mean, 37.9 months; range, 24-56 months) (14). We found the families of 34 (60\%) patients reported that they were adherent with our recommendations for brace use. When all variables are taken into account, adherence to brace use was not found to correlate with annual family income $(\mathrm{P}=0.50)$, educational level of parents $(\mathrm{P}=0.34)$, or marital status of parents $(\mathrm{P}=0.76)$. It should be noted that the majority of the patients' families had annual incomes of less than $\$ 20,000$.
A relapsed deformity was observed in 40 (48\%) of feet; correction was regained by further cast treatment in all cases, and no patient had undergone posteromedial release surgery. Eighteen $(21 \%)$ feet had undergone TATT. Of note, at latest follow-up, all feet were plantigrade and had at least 10 degrees of dorsiflexion. In these early results, we found relapse to be strongly associated with brace adherence; specifically, patients were 2 to 3 times more likely to relapse in non-adherent families.

Ponseti (1) emphasized that evaluation of functional results of clubfoot treatment necessitates follow-up into adult life, noting that "most children with defective feet do not complain; their endurance and activity are boundless". Furthermore, he observed that children were not as limited by joint stiffness and muscle weakness. Consequently, he wrote that clinical outcomes of clubfoot patients "cannot be assessed before at least 5 years of age when relapses become infrequent". The outcome studies having the longest followup to date come from Ponseti's institution $(2,20,32)$. The key findings from these studies have been summarized (Table 1).

To further assess the outcome of treatment in our patients, a follow-up study at our institution evaluated 101 patients (147 clubfeet) treated between July 2006 and April 2012 and was published in 2018 (13). Patients were followed for a minimum of 5 years (mean, 6.8 years; range, 5 to 10.1 years). Initial correction was achieved in all feet. However, only $37 \%$ of families were adherent with the brace protocol throughout treatment; $68 \%$ of patients experienced a relapsed deformity on one or more occasions, and $38 \%$ underwent a TATT. Notwithstanding, utilizing the Dallas criteria (11), $62 \%$ of patients had a good clinical outcome, $38 \%$ had fair outcomes, with none having a poor 
outcome. Using the Roye DSI (12), most families were very satisfied with the function and appearance of the feet. These findings are consistent with those reported for Ponseti's patients (Table 1).

Both the occurrence of one or more relapse(s) and the age at which the brace was discontinued were significantly associated with the outcome according to the Dallas criteria $(\mathrm{P}<0.01)$. Additionally, we found that severity of deformity was associated with outcome according to the Dallas criteria; patients with a fair outcome had higher initial severity scores. Since, as a fair outcome was a plantigrade foot that required a TATT, our findings suggested that patients with a more severe deformity are more likely to undergo a TATT. Ponseti offered a possible explanation for our observation. Rather than due to muscle imbalance, he believed that supination was due to partial medial displacement of the navicular and varus of the calcaneus. $\mathrm{He}$ noted that relapses were common in severe cases of clubfoot for which a full correction of the displaced navicular was not possible. Prior to tendon transfer, he maintained that cast treatment was necessary to bring the forefoot into proper alignment by stretching the ligamentous structures distal to the navicular, allowing lateral displacement and angulation of the cuneiforms and abducting the cuboid into its normal position relative to the calcaneus. Cast treatment also allowed the calcaneus to abduct enough to bring the heel into a normal position. This "spurious" correction, as he referred to it, "is compatible with a fully functional, wellaligned foot" (1).

In summary, we found rates of objectively measured variables-incidence of relapse(s) and rate of TATT surgeries-were compatible to those reported in similar intermediate and long-term studies from Ponseti's institution (Table 1). These similarities were not surprising in that the treatment provided in those studies had changed very little. Ensuring proper brace use remains a challenge; given longer brace use appeared to yield better outcomes. Relapses and TATT surgery are likely to remain common to treatment using the Ponseti method, and these events do not adversely affect the patient function or satisfaction with treatment.

Later in his career, Ponseti $(1,3,24)$ expressed optimism that the rate of relapse may diminish in future patients managed with his method due to modification of his cast technique by overcorrecting the deformity in the last cast, as well as a better understanding of the importance of brace use. However, this hypothesis awaits confirmation by intermediate- or long-term data.

\section{Incidence of relapse and secondary surgery in Ponseti- treated clubfeet}

Ponseti (1) observed that, regardless of the method used to obtain correction, "the clubfoot has a stubborn tendency to relapse". The reported rate of relapsed deformity, and the use of secondary surgical procedures, varies widely in the literature. We performed a systematic review using the Preferred Reporting Items for Systematic Reviews and Meta-Analyses guidelines and found that reported relapse rates following Ponseti treatment, ranged from $3.7 \%$ to $63 \%$ of patients (34). Studies with longer follow-up showed a significant increase in both the rate of relapsed deformity and the percentage of feet requiring a joint sparing surgical procedure as the duration of follow-up increased $(\mathrm{P}<0.05)$. The study also identified four unique definitions of relapse, which certainly influenced the reported rates of relapse and the need for subsequent surgical procedures. Accordingly, a precise definition of relapse should be adopted in future studies to allow more consistent comparison of findings.

While studies with a shorter duration of follow-up have reported more favorable outcomes, we were unable to predict whether those findings were due to later refinements of the Ponseti technique, or whether increased follow-up would result in findings comparable to Ponseti's. Therefore, long-term follow-up studies are needed to settle this issue.

\section{Survivorship analysis of relapsed deformity}

We used Kaplan-Meier survivorship and multivariate regression analysis on a group of 191 clubfoot patients from our clinic to study the probability of relapse over time (35). In this study, 94 patients (49\%) experienced at least one relapse. Overall, the probability of relapse increased from $30 \%$ at age 2 years to $45 \%$ at age 4 years. The probability rose much less-from $45 \%$ to $52 \%$-between ages 4 to 6 years (Figure 1). Of the 85 patients $(44.5 \%)$ whose parents reported adherence to the recommended brace protocol, the probability of relapse was $11 \%$ at 4 years of age rising to $24 \%$ by 6 years of age (Figure 2). In patients whose parents were not adherent to bracing, the probability of relapse was much greater $(72 \%)$ by 4 years. Multivariate regression analysis indicated that parent-reported brace adherence reduced the odds of relapse by nearly 15 times $(\mathrm{P}<0.01)$.

The severity of deformity was highly correlated with the probability of relapse. Specifically, at 4 years of age, patients with a very severe deformity had a $76 \%$ risk of relapse, whereas patients with a moderate deformity had a $42 \%$ risk of relapse (Figure 3). Feet graded as very severe were at least 


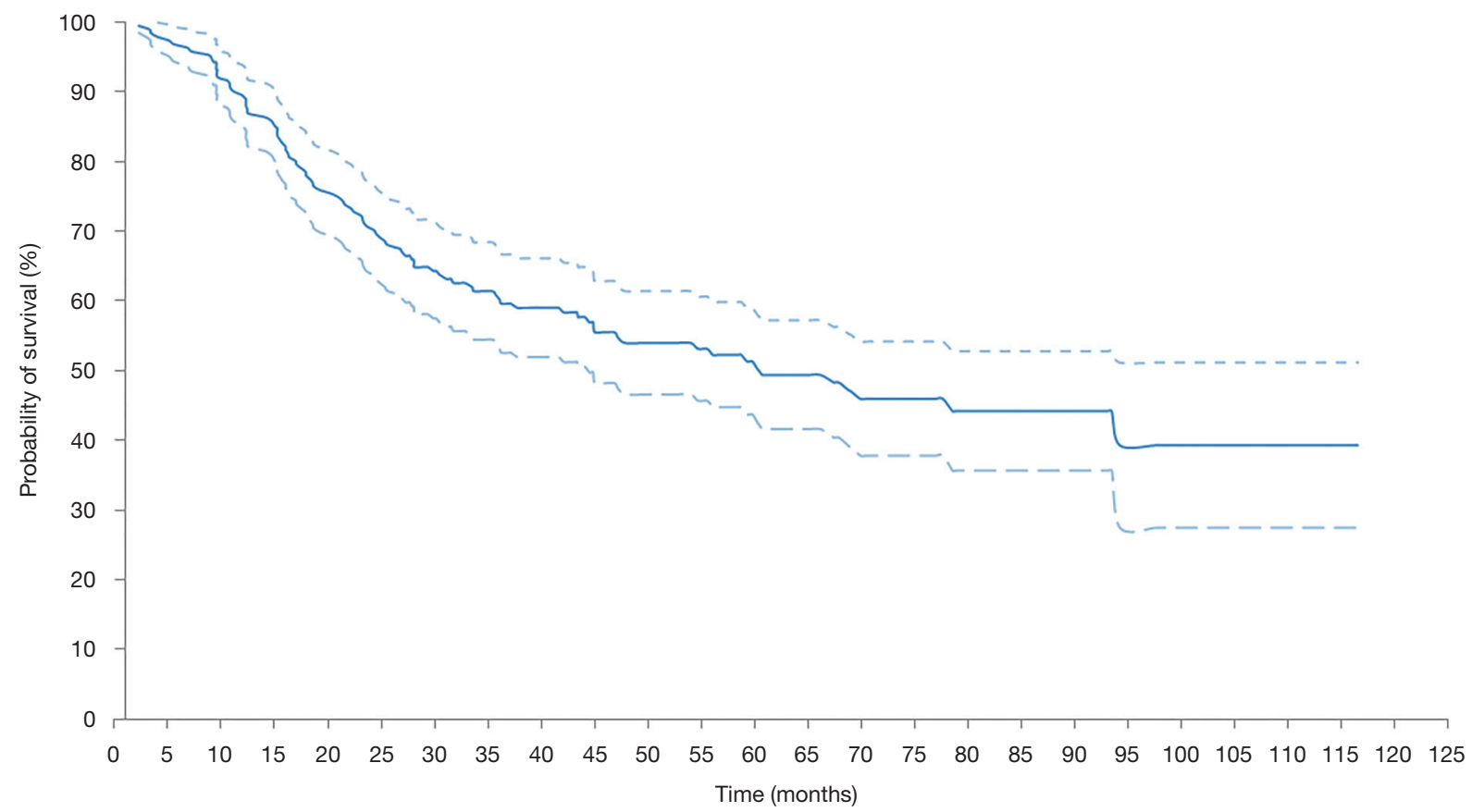

Figure 1 Kaplan-Meier survival plot showing the probability of avoiding a relapse in deformity as a function of patient age during early childhood, along with the $95 \%$ confidence interval depicted with dotted lines [from reference (35)].

Adherence vs. non-adherence

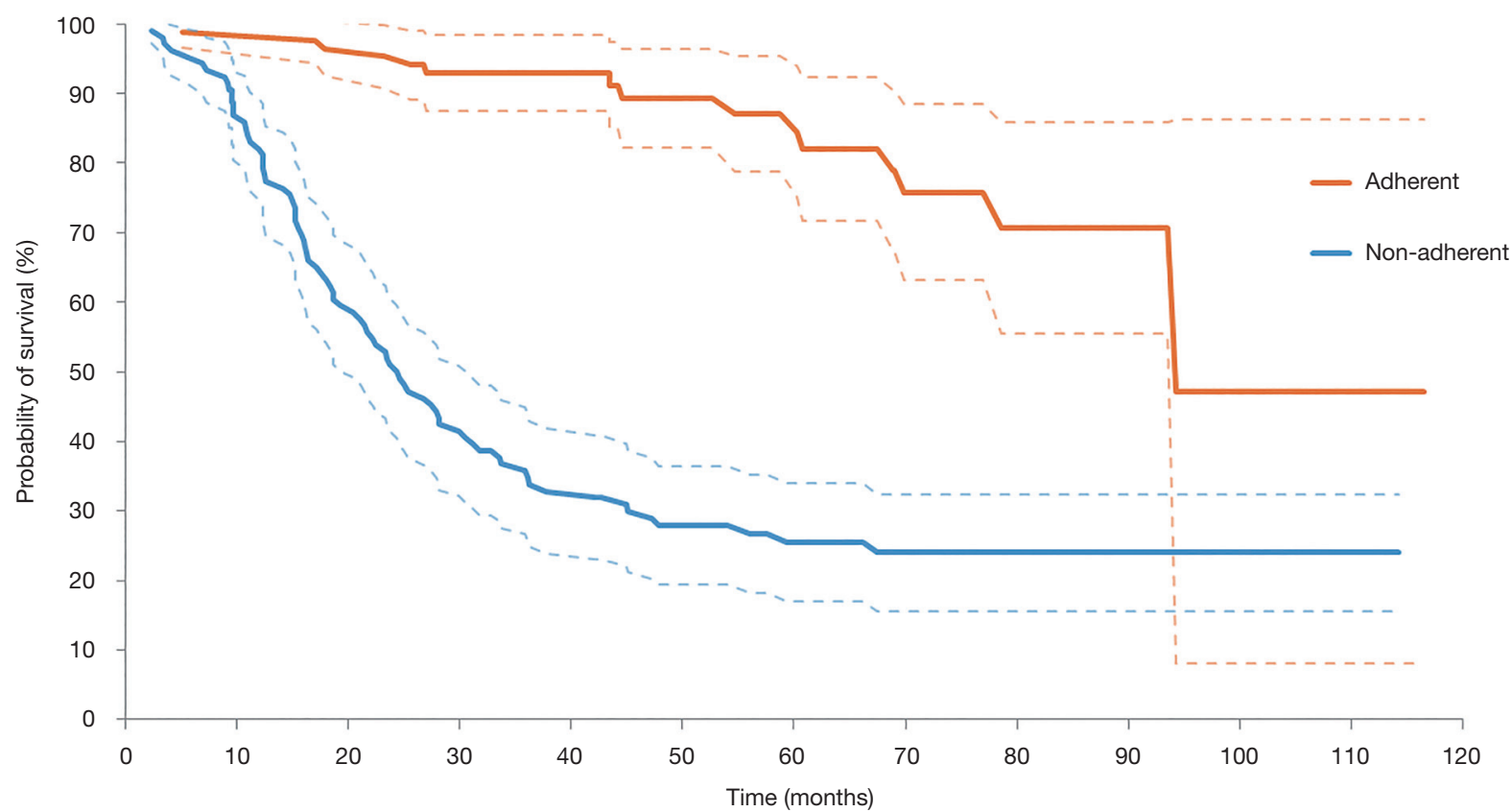

Figure 2 Kaplan-Meier survival plot showing the probability of avoiding a relapse as a function of patient age in patients whose parents were adherent to the prescribed brace protocol in orange, and those who were non-adherent to the brace protocol in blue. The presented data is based on parent-reported adherence or lack of adherence [from reference (35)]. The 95\% confidence intervals are depicted with dotted lines. 


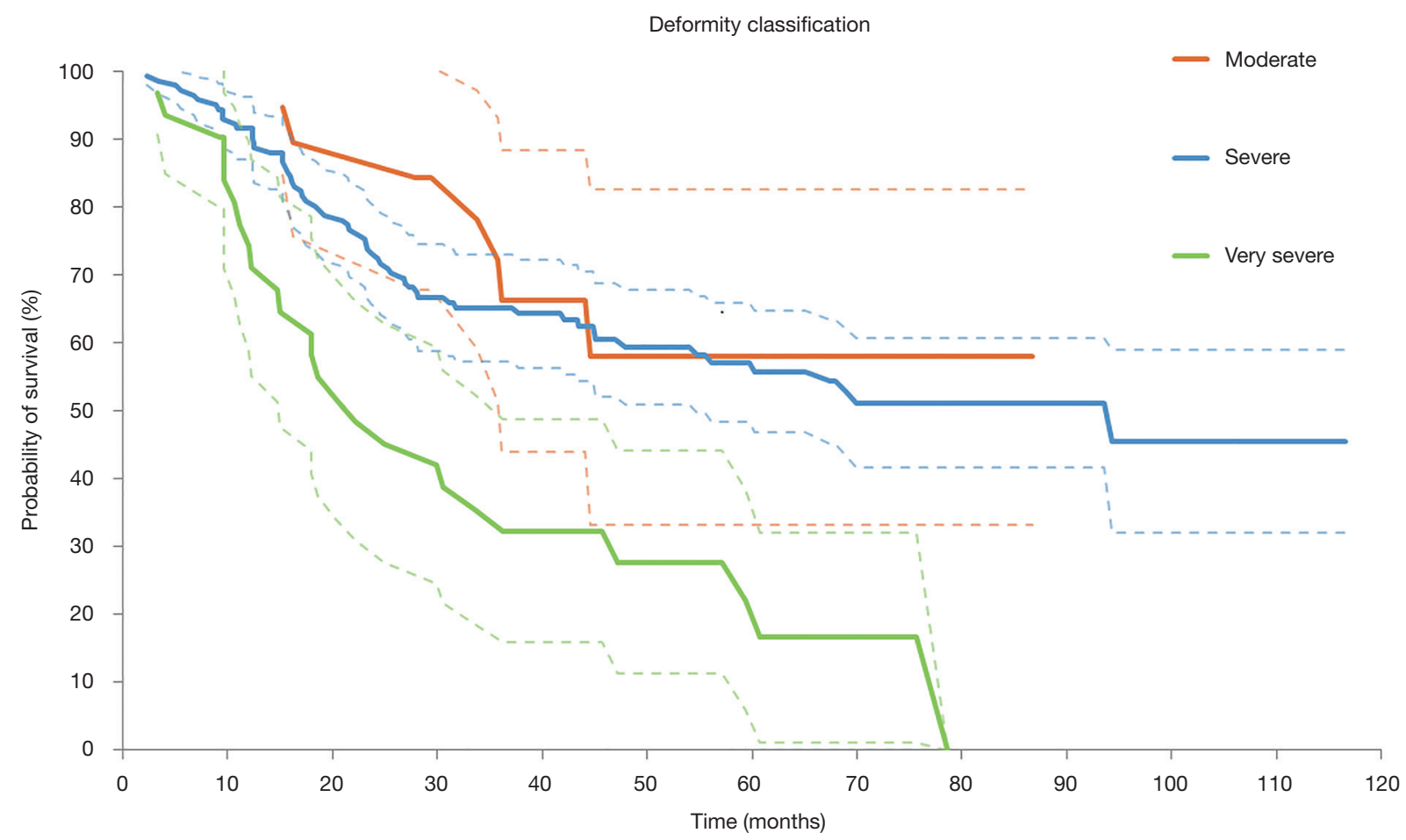

Figure 3 Kaplan-Meier survival plot showing the probability of avoiding a relapse in deformity as a function of patient age during early childhood, shown separately for patients with initial moderate (orange), severe (blue), or very severe (green) deformities as assessed using the Diméglio classification system [from reference (35)]. The $95 \%$ confidence intervals for each deformity classification are depicted with dotted lines.

5.8 times more likely to relapse than those graded moderate or severe $(\mathrm{P}<0.01)$.

Since our findings indicated the probability of relapse rose much less after age 4 years, we concluded that postcorrective bracing should best be continued until age 4 or 5 years to reduce the risk of relapse. Similarly, based on the findings that clubfeet initially graded as very severe were significantly more likely to relapse, we suggested bracing for these patients should be continued to age 5 years.

\section{Survivorship analysis of the need for TATT}

We used Kaplan-Meier survivorship analysis of 137 idiopathic clubfoot patients who were followed for 30 to 98 months; 34 (24.8\%) underwent a TATT (36). Overall, the probability of undergoing a TATT remained below $5 \%$ at 3 years of age but exceeded $15 \%$ by 4 years, increasing progressively to $29 \%$ by 6 years of age (Figure 4). Of note, this number was significantly reduced by family adherence to the post-corrective brace protocol. Specifically, at 6 years of age, patients whose families applied their infant's brace as prescribed, showed only a $19 \%$ probability of having a TATT compared to a $42 \%$ probability in families who did not reliably apply the brace. The study emphasizes the importance of careful brace use to minimize the need for later surgery and the need to counsel families accordingly throughout treatment.

\section{Monitoring brace use}

It is generally agreed that a successful outcome of Ponsetitreated clubfeet requires consistent adherence to bracing on the part of parents. However, nearly all outcome studies have relied on parental reporting to determine how reliably the brace is worn. In a cross-sectional study of 48 patients in four age-based groups, we used wireless sensors to quantify brace use over a 3-month period to assess parental adherence to our brace recommendations (37). We found that the median brace use recorded by the sensors was $62 \%$ of that recommended, and $77 \%$ of that reported by the parents. We also found that patients who wore the brace more than 8 hours per day were less likely to 


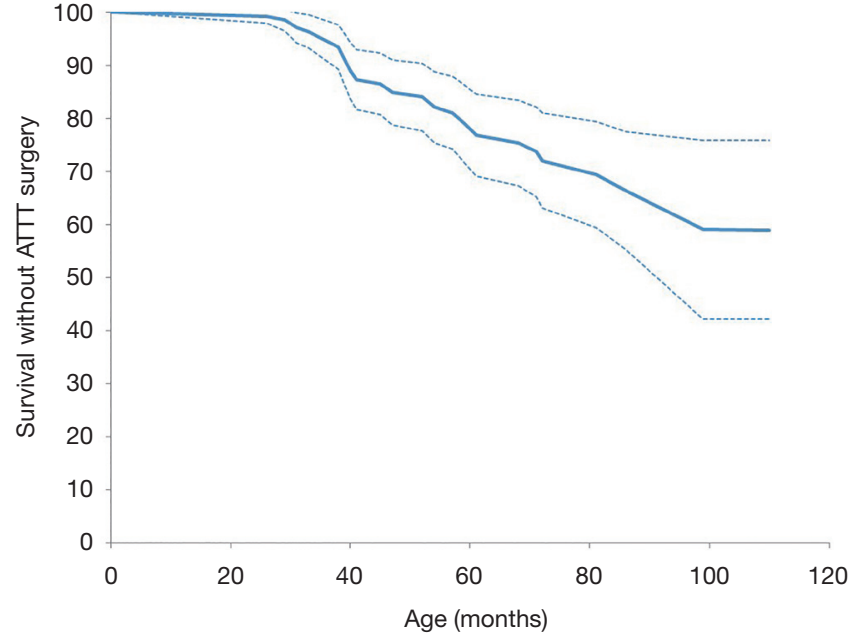

Figure 4 Kaplan-Meier survival plot showing the probability of avoiding the need for a transfer of the tibialis anterior tendon due to relapse in deformity as a function of patient age is shown, along with the $95 \%$ confidence interval depicted with dotted lines [from reference (36)].

experience a relapse than those who wore it less than 5 hours per day. While our study could not determine with sufficient statistical certainty the number of hours - or brace doseneeded to prevent a relapse, the results suggested that brace use of 8 hours or more may be helpful to avoid this problem. In the future, larger studies using improved sensors and data retrieval systems may allow us to routinely monitor all patients and more accurately determine the minimum number of hours of bracing needed to prevent relapse.

\section{When to begin Ponseti treatment}

A clubfoot deformity may be diagnosed on ultrasound or at the time of birth. When a family presents for prenatal counseling or discovers in the newborn nursery that their infant has a clubfoot, the first question they frequently ask is how soon treatment should begin. Ponseti advised that initial treatment should begin as early as possible to take advantage of the more favorable viscoelastic properties of the newborn's connective tissue. This view has been supported by several pediatric and pediatric orthopaedic textbooks. To determine whether patient-related and early treatment variables correlated with the age at which treatment began, we evaluated 176 infants at our clubfoot clinic who had not received prior treatment (38). All patients began treatment by 26 weeks of age (mean, 6.1 weeks; range, 0.3 to 25.9 weeks). We found no influence of the age at onset of treatment and the number of casts required prior to tenotomy, cast skin problems, early brace adherence, brace skin problems, or early relapse $(\mathrm{P} \geq 0.45)$. We did find that cast slippage occurred more often in those patients beginning treatment earlier $(\mathrm{P}=0.05)$. The results of our study indicated that the cast and early brace phases of clubfoot treatment are not appreciably altered by a delay of a few weeks before beginning treatment. This information may be used to allay the concerns of families who may not have immediate access to clubfoot treatment. Specifically, if the mother of a newborn with a clubfoot is having postpartum difficulties, she should not feel pressure to urgently initiate treatment.

\section{Influence of sex and laterality on clubfoot severity}

Idiopathic clubfoot is bilateral in approximately $50 \%$ of cases and has been commonly reported to affect males more often than females. Some investigators have suggested that female patients $(39,40)$ and bilateral patients $(40)$ may represent a more severe phenotype due to underlying genetic mechanisms; however, there were no data to confirm this. Therefore, we evaluated 240 infants at our clubfoot clinic to examine the relationship between sex and laterality on clubfoot severity (41).

Severity of deformity was determined using the Diméglio classification system; correlations were quantified by nonparametric analysis. We found no difference in clubfoot severity due to sex $(\mathrm{P}=0.99)$, but patients with bilateral clubfeet were more likely to have either moderate or very severe deformities compared to those patients with a unilateral clubfoot, who were more likely to have severe deformities $(\mathrm{P}<0.01)$. We concluded that there was no difference in severity due to sex, and that clubfoot occurring in bilateral patients did not have increased severity, but rather presented with a larger range of severity.

These findings may call into question the speculation of previous investigators that female patients and patients with bilateral deformity, may represent a more severe phenotype based on underlying genetic mechanisms. Regardless, sex and laterality of idiopathic clubfoot deformity should not influence treatment decisions.

\section{Influence of generalized joint hypermobility on clubfoot treatment}

Generalized joint hypermobility has long been a suspected 
risk factor for clubfoot. In 1964, Wynne-Davies (42) reported evidence that of an association between generalized joint laxity and clubfoot; a finding later supported by Olshan and colleagues (43). As well, clubfoot has been noted to be a common finding in patients with Ehlers-Danlos syndrome (44) and Loeys-Dietz syndrome (45), both hereditary connective tissue disorders in which joint hypermobility is a common feature. Ponseti suggested that clubfeet in children having "loose ligaments" were less prone to relapses (24). Recently, Cosma et al. (46) suggested that joint hyperlaxity could be a protective factor for relapse; a finding they considered preliminary given the small number of patients in their study.

We sought to determine if generalized joint hypermobility, influenced the Ponseti treatment of clubfoot in a cohort of 57 patients with clubfoot deformity (47). All patients were assigned a Beighton score by a physical therapist. Although we found the Beighton scores were slightly higher in patients who had experienced no relapse, the difference was not significant $(\mathrm{P}=0.10)$. As well, we found no influence of the Beighton score on sex, laterality, initial severity, number of pre-tenotomy casts, the need for tenotomy, or the need for TATT. None of the clubfeet exhibited overcorrection defined as excessive pes planus or hindfoot valgus that required any specific additional treatment. Of note, we found the Beighton scores of our patients were greater compared to reports involving healthy children, supporting an association between increased joint mobility and idiopathic clubfoot. We concluded that the presence of generalized joint hypermobility did not influence the outcome of treatment. The presence of joint laxity should not influence aspects of clubfoot treatment, such as the duration of bracing, or predicting future relapse or the need for surgery.

\section{Influence of Ponseti treatment on independent walking}

At the start of clubfoot treatment, many parents express concern over whether the Ponseti method will affect the motor development of their child, particularly with regard to independent walking. We investigated the age at which infants at our clubfoot clinic achieved independent walking (48). Included were 94 patients with idiopathic clubfoot who were full term at birth, began treatment by 12 weeks of age, and were followed for a minimum of 24 months. Overall, the mean age at which our clubfoot patients began walking independently was $14.5 \pm 2.6$ months. In contrast, the mean age at which healthy patients without clubfoot begin to walk has been reported to be $12.1 \pm 1.8$ (49). By 16.5 months, 71 (75\%) of our clubfoot patients were walking, and by 18 months, 85 (90\%) were walking. Overall, initial severity of deformity had the greatest influence on the onset of walking. Patients with a moderate or severe deformity began walking one month earlier than those with very severe deformities (14.2 vs. 15.8 months; $\mathrm{P}=0.03)$. Further, patients who experienced a relapse prior to walking, began walking 2 months later than those who did not relapse (15.9 vs. 14.2 months; $\mathrm{P}=0.04)$. Compared to previous reports of otherwise healthy infants, we concluded that the parents whose infants are treated using the Ponseti method should expect their child to achieve independent walking approximately 2 months later than infants without a clubfoot. A greater delay may be expected for those patients who have a very severe deformity or those who experience an early relapse.

\section{When is the third cuneiform ossification center large enough for tendon transfer?}

A TATT is an important adjunct to the Ponseti method and is considered in patients who experience a relapse of clubfoot correction $(1,2,20,25)$. The procedure is generally recommended for patients older than 2.5 years of age as it was presumed that by this age, sufficient ossification had occurred in the third cuneiform to allow bone-to tendon healing and avoid damage to the cartilaginous anlage (25). To quantify the ossification process of the lateral cuneiform between 1 and 3 years of age, we measured the length, width, and height of this osseous structure in 43 consecutive patients in our clubfoot clinic with a unilateral idiopathic clubfoot deformity (50). We observed that the dimensionslength, height, and width—on the affected side increased annually, but remained smaller than the corresponding dimensions of the unaffected foot $(\mathrm{P}<0.01)$. We concluded that the ossific center of the lateral cuneiform may not be sufficiently large to accommodate a tendon transfer before the age of 3 years. After the age of 3 years, the volume of ossification of the bone is sufficient to allow a better chance of bone-to-tendon healing, and less risk of damage to the cartilaginous anlage.

\section{Conclusions}

The Ponseti method has become the standard of care to manage idiopathic clubfoot deformity. The method describes not only how to achieve initial correction of 
the deformity, but also how to manage relapses that will inevitably occur in many patients. Complete anatomic correction of the foot should not be expected; however, the resulting osseous alignment and range of motion that can be achieved is sufficient to allow normal activities. Perhaps most importantly, the Ponseti method largely eliminates the need for open joint surgery which has the propensity to lead to stiffness, limited function, and pain in adult life.

Intermediate-term outcome results from our institution have shown that while relapses and TATT surgery remain common to the Ponseti method, these events do not adversely affect overall patient function or satisfaction. While our data suggest the FAO should be worn at least until age 4 years, encouraging the family to adhere to the recommended duration of post-corrective brace use remains a challenge. The importance of bracing to a successful outcome must be stated early and often during interactions with the family. In addition, a patient approach is needed when relapses arise to encourage the family to accept the need for further cast treatment or tendon transfer.

Some future directions for further enhancement of the Ponseti method include the need to accurately assess brace adherence and to determine the dose and duration of bracing necessary for an optimal outcome. Specifically, the current bracing protocol is not customized based on a particular patient's needs or prognosis. This is due to the fact that insufficient knowledge exists on the relationship between bracing protocol and outcome. Future studies need to address this shortcoming using well controlled, large scale studies with objective measurements combined with detailed clinical and functional outcome assessment. More intermediate- and long-term outcome studies are needed to help clarify whether any recent modifications to the original technique will lead to better outcomes, fewer relapses, and a lessened dependence on tendon transfer surgery.

\section{Acknowledgments}

We thank our patients and their families for their participation in our clinical research studies. We thank our pediatric orthopaedic fellows and research assistants who participated in our research studies at the Orthopaedic Institute for Children and the J. Vernon Luck, Sr., M.D., Orthopaedic Research Center.

Funding: Funding was provided in part by the Orthopaedic Hospital Doctors' Education and Research Fund and the 2014 Pediatric Orthopaedic Society of North America Research Grant.

\section{Footnote}

Provenance and Peer Review: This article was commissioned by the Guest Editors (Federico Canavese and Alain Dimeglio) for the series "Clubfoot" published in Annals of Translational Medicine. The article has undergone external peer review.

Peer Review File: Available at http://dx.doi.org/10.21037/ atm-20-7774

Conflicts of Interest: All authors have completed the ICMJE uniform disclosure form (available at http://dx.doi. org/10.21037/atm-20-7774). The series "Clubfoot" was commissioned by the editorial office without any funding or sponsorship. The authors have no other conflicts of interest to declare.

Ethical Statement: The authors are accountable for all aspects of the work in ensuring that questions related to the accuracy or integrity of any part of the work are appropriately investigated and resolved.

Open Access Statement: This is an Open Access article distributed in accordance with the Creative Commons Attribution-NonCommercial-NoDerivs 4.0 International License (CC BY-NC-ND 4.0), which permits the noncommercial replication and distribution of the article with the strict proviso that no changes or edits are made and the original work is properly cited (including links to both the formal publication through the relevant DOI and the license). See: https://creativecommons.org/licenses/by-nc-nd/4.0/.

\section{References}

1. Ponseti IV. Congenital clubfoot. Fundamentals of treatment. New York: Oxford University Press, 1996.

2. Ponseti IV, Smoley EN. Congenital clubfoot: the results of treatment. J Bone Joint Surg Am 1963;45:261-75.

3. Morcuende JA, Dolan LA, Dietz FR, et al. Radical reduction in the rate of extensive corrective surgery for clubfoot using the Ponseti method. Pediatrics 2004;113:376-80.

4. Hosseinzadeh P, Kiebzak GM, Dolan L, et al. Management of clubfoot relapses with the Ponseti method: results of a survey of the POSNA members. J Pediatr Orthop 2019;39:38-41.

5. Ponseti IV. Treatment of congenital club foot. J Bone Joint Surg Am 1992;74:448-54. 
6. Ippolito E, Ponseti IV. Congenital club foot in the human fetus. A histological study. J Bone Joint Surg Am 1980;62:8-22.

7. Dobbs MB, Nunley R, Schoenecker PL. Long-term follow-up of patients with clubfeet treated with extensive soft-tissue release. J Bone Joint Surg Am 2006;88:986-96.

8. Zionts LE, Zhao G, Hitchcock K, et al. Has the rate of extensive surgery to treat idiopathic clubfoot declined in the United States? J Bone Joint Surg Am 2010;92:882-9.

9. Zionts LE, Sangiorgio SN, Ebramzadeh E, et al. The current management of idiopathic clubfoot revisited: results of a survey of the POSNA membership. J Pediatr Orthop 2012;32:515-20.

10. Diméglio A, Bensahel H, Souchet P, et al. Classification of clubfoot. J Pediatr Orthop B 1995;4:129-36.

11. Richards BS, Faulks S, Rathjen KE, et al. A comparison of two nonoperative methods of idiopathic clubfoot correction: the Ponseti method and the French functional (physiotherapy) method. J Bone Joint Surg Am 2008;90:2313-21.

12. Roye BD, Vitale MG, Gelijns AC, et al. Patient-based outcomes after clubfoot surgery. J Pediatr Orthop 2001;21:42-9.

13. Zionts LE, Ebramzadeh E, Morgan RD, et al. Sixty years on: Ponseti method for clubfoot treatment produces high satisfaction despite inherent tendency to relapse. J Bone Joint Surg Am 2018;100:721-8.

14. Zionts LE, Frost N, Kim R, et al. Treatment of idiopathic clubfoot: experience with the mitchell-ponseti brace. J Pediatr Orthop 2012;32:706-13.

15. Coss HS, Hennrikus WL. Parent satisfaction comparing two bandage materials used during serial casting in infants. Foot Ankle Int 1996;17:483-6.

16. Hui C, Joughin E, Nettel-Aguirre A, et al. Comparison of cast materials for the treatment of congenital idiopathic clubfoot using the Ponseti method: a prospective randomized controlled trial. Can J Surg 2014;57:247-53.

17. Göksan SB, Bursali A, Bilgili F, et al. Ponseti technique for the correction of idiopathic clubfeet presenting up to 1 year of age. A preliminary study in children with untreated or complex deformities. Arch Orthop Trauma Surg 2006;126:15-21.

18. Janicki JA, Narayanan UG, Harvey BJ, et al. Comparison of surgeon and physiotherapist-directed Ponseti treatment of idiopathic clubfoot. J Bone Joint Surg Am 2009;91:1101-8.

19. Mosca VS. The foot. In: Weinstein SL, Flynn JM. editors. Lovell and Winter's Pediatric Orthopaedics. 7th ed.
Philadelphia: Lippincott Williams \& Wilkins; 2013:1445.

20. Laaveg SJ, Ponseti IV. Long-term results of treatment of congenital club foot. J Bone Joint Surg Am 1980;62:23-31.

21. Iravani M, Chalabi J, Kim R, et al. Propofol sedation for infants with idiopathic clubfoot undergoing percutaneous tendoachilles tenotomy. J Pediatr Orthop 2013;33:59-62.

22. Bor N, Katz Y, Vofsi O, et al. Sedation protocols for Ponseti clubfoot Achilles tenotomy. J Child Orthop 2007;1:333-5.

23. Parada SA, Baird GO, Auffant RA, et al. Safety of percutaneous tendoachilles tenotomy performed under general anesthesia on infants with idiopathic clubfoot. J Pediatr Orthop 2009;29:916-9.

24. Ponseti IV. Relapsing clubfoot: causes, prevention, and treatment. Iowa Orthop J 2002;22:55-6.

25. Dietz FR. Treatment of a recurrent clubfoot deformity after initial correction with the Ponseti technique. Instr Course Lect 2006;55:625-9.

26. Hosseinzadeh P, Kelly DM, Zionts LE. Management of the relapsed clubfoot following treatment using the Ponseti method. J Am Acad Orthop Surg 2017;25:195-203.

27. Ezra E, Hayek S, Gilai AN, et al. Tibialis anterior tendon transfer for residual dynamic supination deformity in treated club feet. J Pediatr Orthop B 2000;9:207-11.

28. Farsetti P, Caterini R, Mancini F, et al. Anterior tibial tendon transfer in relapsing congenital clubfoot: longterm follow-up study of two series treated with a different protocol. J Pediatr Orthop 2006;26:83-90.

29. Thompson GH, Hoyen HA, Barthel T. Tibialis anterior tendon transfer after clubfoot surgery. Clin Orthop Relat Res 2009;467:1306-13.

30. Jeans KA, Tulchin-Francis K, Crawford L, et al. Plantar pressures following anterior tibialis tendon transfers in children with clubfoot. J Pediatr Orthop 2014;34:552-8.

31. Mindler GT, Kranzl A, Radler C. Normalization of forefoot supination after tibialis anterior tendon transfer for dynamic clubfoot recurrence. J Pediatr Orthop 2020;40:418-24.

32. Cooper DM, Dietz FR. Treatment of idiopathic clubfoot. A thirty-year follow-up note. J Bone Joint Surg Am 1995;77:1477-89.

33. Holt JB, Oji DE, Yack HJ, et al. Long-term results of tibialis anterior tendon transfer for relapsed idiopathic clubfoot treated with the Ponseti method: a follow-up of thirty-seven to fifty-five years. J Bone Joint Surg Am 2015;97:47-55.

34. Thomas HM, Sangiorgio SN, Ebramzadeh E, et al. Relapse rates in patients with clubfoot treated using the 
Ponseti method increase with time: a systematic review. JBJS Rev 2019;7:e6.

35. Sangiorgio SN, Ebramzadeh E, Morgan RD, et al. The timing and relevance of relapsed deformity in patients with idiopathic clubfoot. J Am Acad Orthop Surg 2017;25:536-45.

36. Zionts LE, Jew MH, Bauer KL, et al. How many patients who have a clubfoot treated using the Ponseti method are likely to undergo a tendon transfer? J Pediatr Orthop 2018;38:382-7.

37. Sangiorgio SN, Ho NC, Morgan RD, et al. The objective measurement of brace-use adherence in the treatment of idiopathic clubfoot. J Bone Joint Surg Am 2016;98:1598-605.

38. Zionts LE, Sangiorgio SN, Cooper SD, et al. Does clubfoot treatment need to begin as soon as possible? J Pediatr Orthop 2016;36:558-64.

39. Goldstein RY, Seehausen DA, Chu A, et al. Predicting the need for surgical intervention in patients with idiopathic clubfoot. J Pediatr Orthop 2015;35:395-402.

40. Kruse LM, Dobbs MB, Gurnett CA. Polygenic threshold model with sex dimorphism in clubfoot inheritance: the Carter effect. J Bone Joint Surg Am 2008;90:2688-94.

41. Zionts LE, Jew MH, Ebramzadeh E, et al. The influence of sex and laterality on clubfoot severity. J Pediatr Orthop 2017;37:e129-33.

42. Wynne-Davies R. Family studies and the cause of congenital club foot. Talipes equinovarus, talipes calcaneo- valgus and metatarsus adductus. J Bone Joint Surg Br 1964;46:445-63.

43. Olshan AF, Schroeder JC, Alderman BW, et al. Joint laxity and the risk of clubfoot. Birth Defects Res A Clin Mol Teratol 2003;67:585-90.

44. Beighton P, Horan F. Orthopaedic aspects of the EhlersDanlos syndrome. J Bone Joint Surg Br 1969;51:444-53.

45. Erkula G, Sponseller PD, Paulsen LC, et al. Musculoskeletal findings of Loeys-Dietz syndrome. J Bone Joint Surg Am 2010;92:1876-83.

46. Cosma DI, Corbu A, Nistor DV, et al. Joint hyperlaxity prevents relapses in clubfeet treated by Ponseti methodpreliminary results. Int Orthop 2018;42:2437-42.

47. Beck JJ, Nazif MA, Sangiorgio SN, et al. Does generalized joint hypermobility influence the Ponseti treatment of clubfoot patients? J Pediatr Orthop B 2021;30:66-70.

48. Zionts LE, Packer DF, Cooper S, et al. Walking age of infants with idiopathic clubfoot treated using the ponseti method. J Bone Joint Surg Am 2014;96:e164.

49. WHO Multicentre Growth Reference Study Group. WHO Motor Development Study: windows of achievement for six gross motor development milestones. Acta Paediatr Suppl 2006;450:86-95.

50. Lang PJ, Avoian T, Sangiorgio SN, et al. Quantification of the ossification of the lateral cuneiform in the feet of young children with unilateral congenital talipes equinovarus. Bone Joint J 2017;99-B:1109-14.
Cite this article as: Zionts LE, Ebramzadeh E, Sangiorgio SN. Objective analysis of intermediate-term outcome of the Ponseti technique: a review of the experience from Los Angeles. Ann Transl Med 2021;9(13):1101. doi: 10.21037/atm-20-7774 